\title{
Overview of Diffraction at the LHC
}

\author{
S. Navin for the ALICE, ATLAS and CMS collaborations
}

School of Physics and Astronomy, University of Birmingham, Edgbaston, Birmingham, B15 2TT, United Kingdom

DOI: http://dx.doi.org/10.5689/UA-PROC-2010-09/29

\begin{abstract}
This is a first look at diffraction with $p p$ collisions at the LHC. The observation of inclusive diffraction at the LHC with the CMS detector at $\sqrt{s}=900 \mathrm{GeV}$ and $2360 \mathrm{GeV}$ is presented. At $\sqrt{s}=7 \mathrm{TeV}$ the multiplicity, pseudorapidity and transverse momentum distributions of a sample enhanced in diffractive interactions in ATLAS are presented. These measurements are presented along with a comparison with predictions of the PYTHIA and PHOJET Monte Carolo event generators.
\end{abstract}

\section{Introduction}

The total $p p$ cross section is the sum of the elastic and inelastic cross sections. Inelastic events can be diffractive or non-diffractive (ND). In ND events, there is an exchange of quantum numbers between the two interacting systems, while in diffractive events, a colour singlet called a Pomeron is exchanged. In QCD, the Pomeron is regarded as a colourless and flavourless multiple gluon [1] or a glueball exchange. The emitted Pomeron can interact with the proton, breaking it up into a group of final-state hadrons. If one of the incoming protons breaks up, the event is single diffractive (SD) and if both the incoming protons break up, the event is double diffractive (DD). A consequence of a colourless Pomeron exchange is that the two final-state hadrons or diffractive masses have a large rapidity gap (LRG) between them.

The characteristic appearance of rapidity gaps in diffractive events is something experimentalists can exploit. However, soft rescattering between the protons fill the rapidity gap and reduce the visible diffractive cross section. These rapidity gap survival probabilities [2] are energy dependent and must be taken into account. Hence it is experimentally challenging to classify diffractive events. The results that follow do not have proton tagging and rely on identifying rapidity gaps.

Different models predict different behaviour for diffractive cross sections and kinematics. The Monte Carlo (MC) generators used in these analyses are PYTHIA6 [3], PYTHIA8 [4] and PHOJET [5]. While both PYTHIA6 and PYTHIA8 have the same cross section modelling, PYTHIA6 lacks a hard diffractive component.

Results are presented for the experiments ATLAS[6] and CMS[7]. A brief description of the detectors that are used for these analyses is included. 


\section{Diffraction in ATLAS}

The ATLAS detector [8] is a general purpose detector that covers nearly full solid angle around the interaction point. The inner tracking detectors cover the pseudorapidity range of $|\eta|<2.5$ and include the silicon pixel detector, a silicon microstrip detector and a transition radiation tracker, arranged in concentric layers. These detectors are placed in a $2 T$ magnetic field provided by the inner solenoid magnet and help in the resolution and identification of charged tracks. The Minimum Bias Trigger Scintillator (MBTS) detectors located at $\pm z=3.56 \mathrm{~m}$ on the front of the endcap calorimeter are segmented into eight sectors in azimuth. They consist of two rings covering a pseudorapidity range of $2.09<|\eta|<2.82$ and $2.82<|\eta|<3.84$.

\subsection{Single-sided events}

In this study, events at $\sqrt{s}=7 \mathrm{TeV}$ with at least one track with $p_{T}>500 \mathrm{MeV}$ and $|\eta|<2.5$ that have hits on at least one side of the MBTS detector are selected - $N_{\text {any }}$. Such a sample consists of about 12-20\% diffractive events. A sub-sample of single-sided events, which is greatly enhanced in diffractive events is when there are hits in only one side of the MBTS detector $N_{s s}$. Such a sub-sample consists of about $85-98 \%$ diffractive events, depending on which MC model is used. The MC samples used are PYTHIA6, PYTHIA8 and PHOJET with default settings. Central diffraction in PHOJET is neglected.

The results presented are not corrected for detector effects.

\subsubsection{Fraction of single-sided events}

Once this sample and sub-sample are selected, the quantity $R_{s s}$ is defined as the ratio of $N_{s s}$ to $N_{\text {any }}$, given by Eqn. 1 .

$$
R_{s s}=\frac{A_{s s}^{D} \sigma_{D}+A_{s s}^{N D} \sigma_{N D}}{A_{a n y}^{D} \sigma_{D}+A_{a n y}^{N D} \sigma_{N D}}
$$

In Eqn. $1 A_{s s}$ and $A_{a n y}$ are the acceptance for $N_{s s}$ and $N_{a n y}$ respectively and $\sigma_{D}$ refers to the combined cross section for SD and DD components.

The value of $R_{s s}$ calculated from data is $[4.52 \pm 0.02$ (stat.) \pm 0.61 (syst.)]\% where the systematic uncertainty arises from the tracking efficiency and MBTS selection efficiency. Data agree well with the predictions of PYTHIA6 (4.01\%) and PYTHIA8 (5.11\%) while the PHOJET prediction $(2.83 \%)$ falls short by $70 \%$ corresponding to $2 \sigma$. These values are seen in Fig. 1 . The data are shown as the horizontal line and the uncertainty as the (yellow) band. The default fractions for cross section used by the MC generators are indicated by the circular markers. While keeping the ratio of SD to DD cross sections fixed to the MC generator predictions, the ratio of diffractive to ND cross sections is varied. The figure shows that the diffractive contribution in PHOJET needs to be increased from $20 \%$ to $30 \%$ to describe data.

\subsubsection{Track distributions}

Presented here are track and event distributions of the single-sided sub-sample where $N_{e v}=N_{s s}$, $p_{T}$ is the transverse momentum of the track, $\eta$ is the pseudorapidity of the track, $n_{t r k}$ is the number of selected tracks per event, $N_{t r k}$ is the total number of selected tracks and $\Delta \eta$ is the size of the gap, i.e. the absolute value of the difference in pseudorapidity between the edge of 


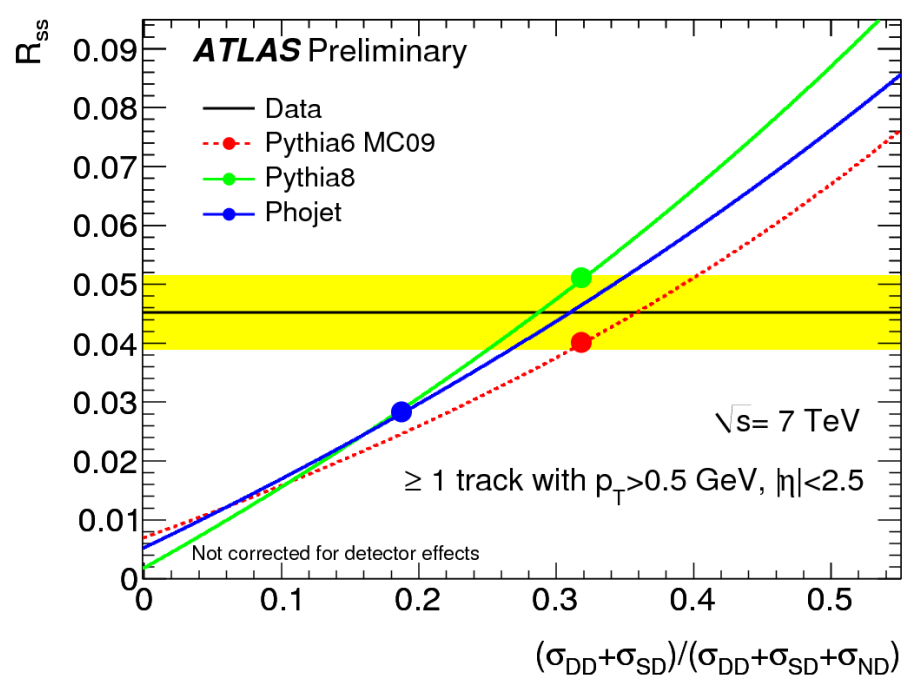

Figure 1: $R_{s s}$ at different fractions of diffractive events.

the MBTS detector with no hits and the track. For SD and DD events, this variable is expected to peak at large $\Delta \eta$.

Figures 2 and 3 show the track $\eta$ and multiplicity respectively. Both these distributions are well modelled by PYTHIA8 and PHOJET. Fig. 4 shows that the track $p_{T}$ distribution in PHOJET agrees very well with data. Fig. 5 shows a distribution of $\Delta \eta$. Although at low $\Delta \eta$ all three MC generators describe data well, at high $\Delta \eta$, where diffraction dominates, PYTHIA6 underestimates the rate of tracks. The bottom plots show the ratio of MC to data, where the (blue) band indicates the quadratic sum of the statistical and systematic uncertainties.

Plots for individual process types in each of the three MC generators can be found in reference [6].

\section{Diffraction in CMS}

The CMS detector [9] is also a general purpose detector with a superconducting solenoid. Enclosed in this field volume are the silicon pixel and strip trackers, the crystal electromagnetic calorimeter (ECAL) and brass-scintillator hadronic calorimeter (HCAL). The forward hadronic calorimeter (HF) covers a pseudorapidity range of $2.9<|\eta|<5.2$. The Beam Scintillator Counters (BSC) and the Beam Pick-up Timing Experiment (BPTX) devices were used to trigger the CMS readout.

An offline selection to maximise the acceptance of SD events required a BPTX signal from both the beams passing the interaction point in conjunction with a signal in either of the BSC devices.

Data are compared with predictions from PYTHIA6 and PHOJET MC generators. 


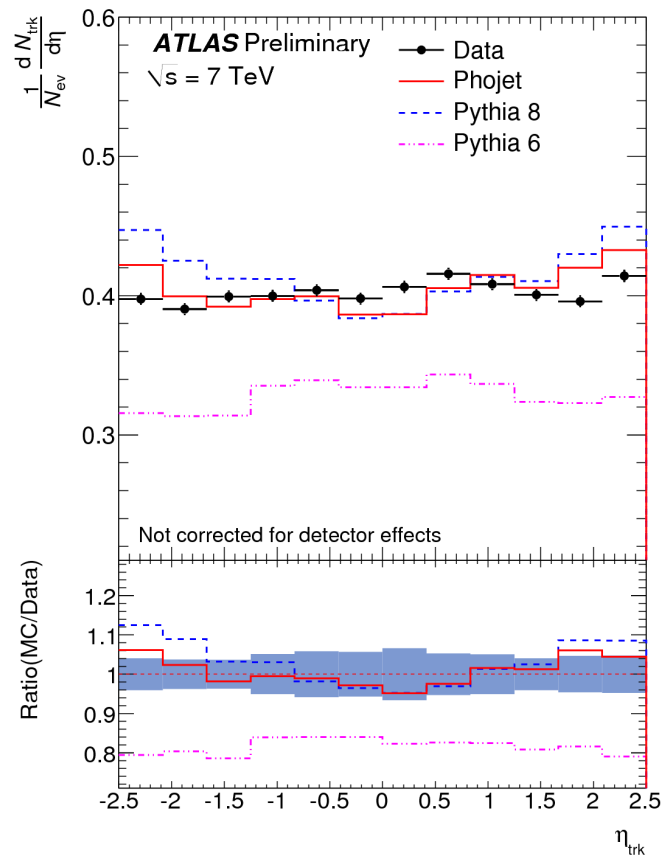

Figure 2: Eta distribution.

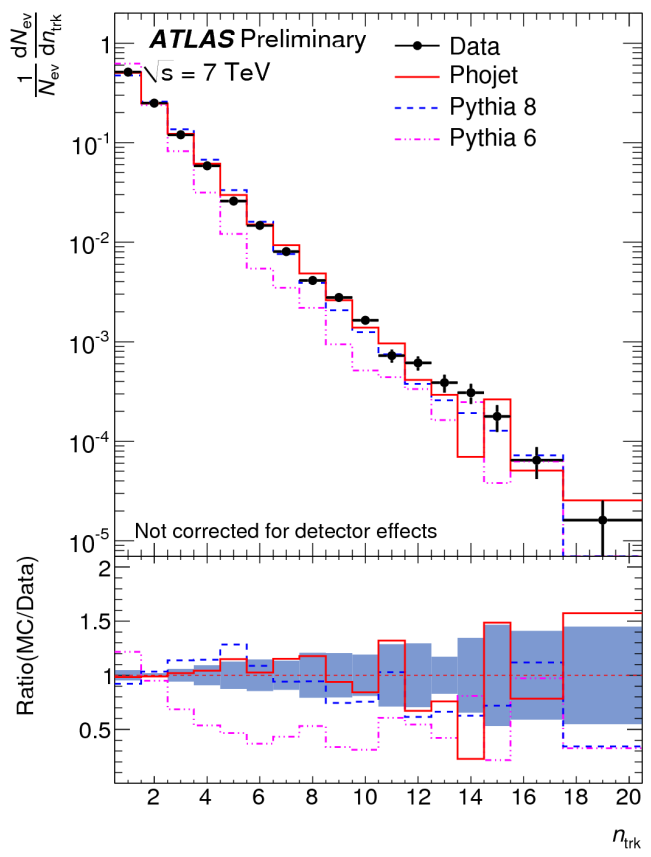

Figure 3: Multiplicity distribution.

\subsection{Diffractive component of data}

Selected events are plotted as a function of the energy deposited in the $\operatorname{HF}\left(E_{H F}\right)$ and the multiplicity of the towers above threshold in HF $\left(N_{H F}\right)$. Figures 6 and 7 show the distributions of accepted events as a function of $E_{H F+}$ and $N_{H F+}$, the energy and multiplicity in the forward rapidities of the $\mathrm{HF}$ at $\sqrt{s}=900 \mathrm{GeV}$. These distributions are uncorrected for detector effects. The MC predictions are normalised to the data. The (yellow) bands illustrate the effect of a $10 \%$ energy scale uncertainty in the calorimeters and is a first estimate of the systematic uncertainty. Diffractive events appear as a peak in the zero-bin of $E_{H F}$ and cluster at zero multiplicities in $N_{H F}$, reflecting the presence of a LRG extending over HF.

PYTHIA describes the ND part of data better than PHOJET.

\subsubsection{Enhancing diffraction}

The diffractive component in the data is enhanced by applying a cut to the HF energy sum. Figs. 8 and 9 show the $E_{H F+}$ and $N_{H F+}$ distributions for events with an energy cut $E_{H F-}<$ $8 \mathrm{GeV}$ for data at $\sqrt{s}=900 \mathrm{GeV}$. This cut enhances the selection of SD events with a LRG over HF-.

PHOJET is in better agreement with data and gives a better description of high-mass diffrac- 


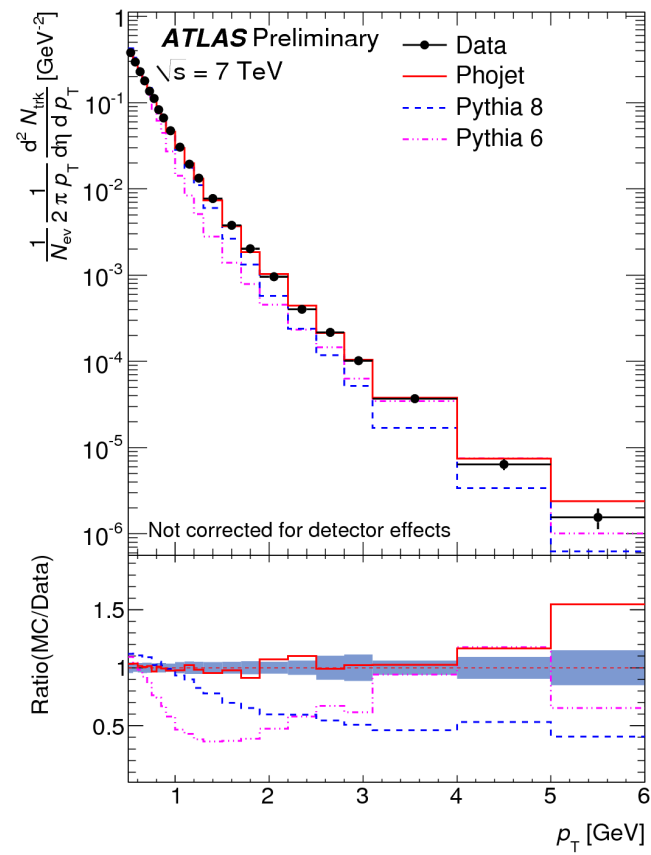

Figure 4: $p_{T}$ distribution.

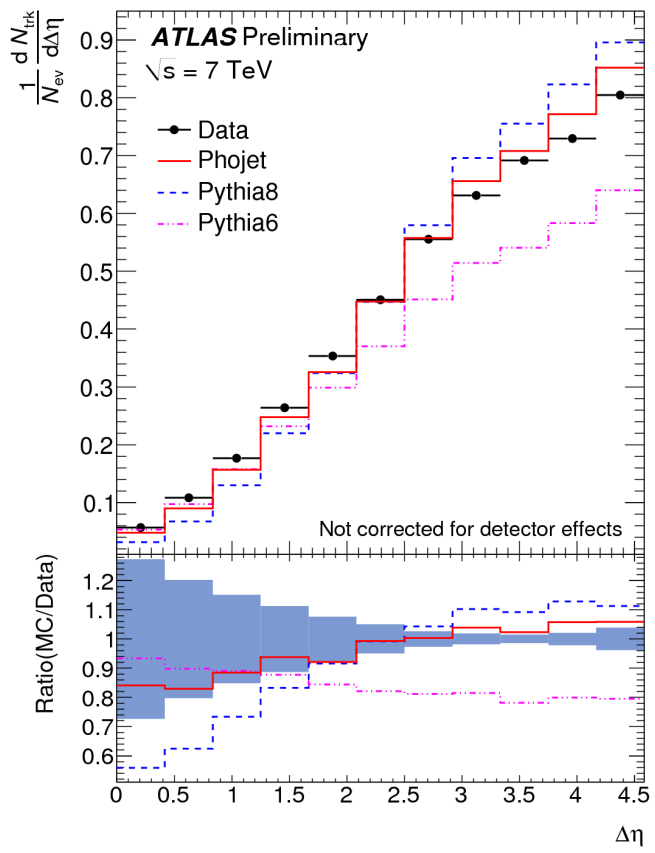

Figure 5: $\Delta \eta$ distribution.

tion, at large values of $E_{H F}$ and $N_{H F}$.

Similar distributions for negative rapidities (HF-) are presented in reference [7]. Also presented are distributions at $\sqrt{s}=2360 \mathrm{GeV}$. At both energies a diffractive contribution is seen.

\section{Conclusions and outlook}

Measurements on data enhanced in diffractive component are made by selecting events with a large rapidity gap in one direction. CMS results are presented on data at $\sqrt{s}=900 \mathrm{GeV}$ and $\sqrt{s}=2360 \mathrm{GeV}$ while ATLAS has presented results at $\sqrt{s}=7 \mathrm{TeV}$. Data have been compared with predictions from the PYTHIA and PHOJET MC generators. PYTHIA models the rate of SD events well. While PHOJET and PYTHIA8 reproduce the diffractive component more accurately, PYTHIA6 gives a better description of the ND component of the data.

\section{Acknowledgments}

Thanks to colleagues on ALICE, ATLAS and CMS, especially, Hannes Jung, Emily Nurse, Roman Lietava, Cristina Lazzeroni and Orlando Villalobos Bailly. 


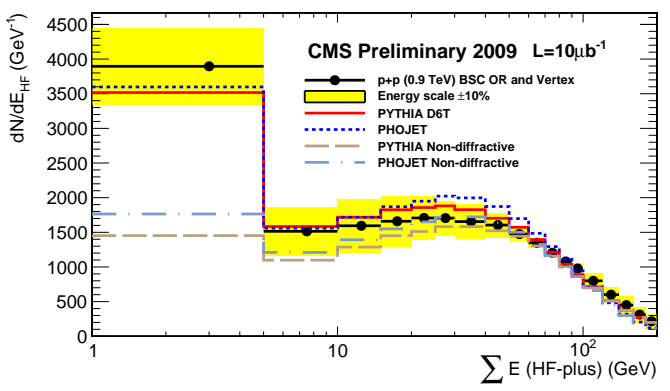

Figure 6: Distribution of accepted events as a function of $E_{H F+}$.

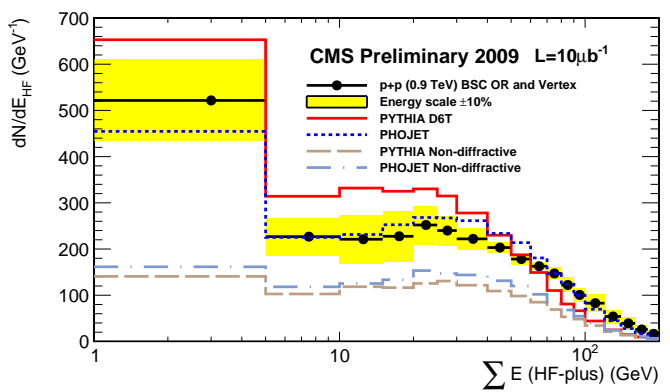

Figure 8: Distribution of accepted events as a function of $E_{H F+}$.

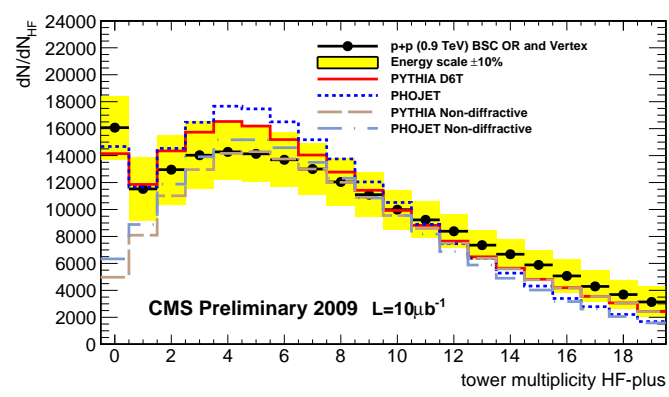

Figure 7: Distribution of accepted events as a function of $N_{H F+}$.

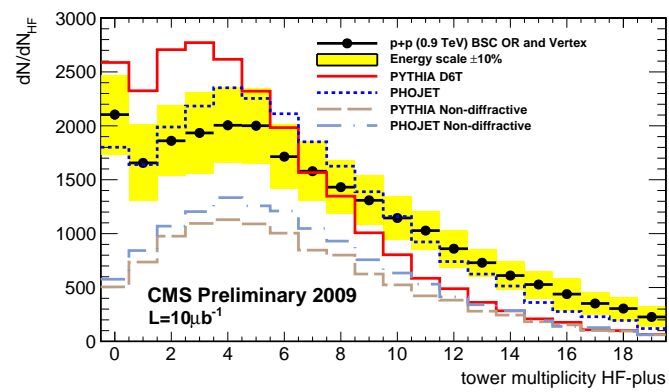

Figure 9: Distribution of accepted events as a function of $N_{H F+}$.

\section{References}

[1] J. R. Forshaw and D. A. Ross, Quantum Chromodynamics and the Pomeron, CUP (1997), p17.

[2] J. D. Bjorken, Phys. Rev. D 47 (1993) 101;

A. B. Kaidalov, V. A. Khoze, A. D. Martin and M. G. Ryskin, Eur. Phys. J. C 21 (2001) 521 [arXiv:hep$\mathrm{ph} / 0105145]$.

[3] T. Sjöstrand, S. Mrenna and P. Skands, JHEP05 (2006) 026 [arXiv:hep-ph/0603175].

[4] T. Sjöstrand, S. Mrenna and P. Skands, Comput. Phys. Comm. 178 (2008) 852;

S. Navin, Diffraction in PYTHIA, arXiv:hep-ph/1005.3894v1.

[5] R. Engel, Z. Phys. C 66 (1995) 203;

R. Engel, J. Ranft, S Roesler, Phys. Rev. D 54 (1996) 4244.

[6] The ATLAS Collaboration, Studies of Diffractive Enhanced Minimum Bias Events in ATLAS, ATLASCONF-2010-048 (2010).

[7] CMS Collaboration, Observation of diffraction in proton-proton collisions at 900 and $2360 \mathrm{GeV}$ centre-ofmass energies at the LHC, PAS-FWD-10-001 (2010).

[8] The ATLAS collaboration, JINST 3 (2008) S08033.

[9] R. Adolphi et al., [CMS Collaboration], JINST 3 (2008) S08004. 